\title{
NONLINEAR VIBRATIONS OF COMPLETELY RESONANT WAVE EQUATIONS
}

\author{
MASSIMILIANO BERTI \\ Dipartimento di Matematica e Applicazioni R. Caccioppoli \\ Università Federico II di Napoli \\ Via Cintia, Monte S. Angelo, 80126, Napoli, Italy \\ E-mail:m.berti@unina.it
}

\begin{abstract}
We present recent existence results of small amplitude periodic and quasi-periodic solutions of completely resonant nonlinear wave equations. Both infinite-dimensional bifurcation phenomena and small divisors difficulties occur. The proofs rely on bifurcation theory, NashMoser implicit function theorems, dynamical systems techniques and variational methods.
\end{abstract}

1. Free vibrations. We consider first completely resonant autonomous wave equations like

$$
\left\{\begin{array}{l}
u_{t t}-u_{x x}+f(x, u)=0 \\
u(t, 0)=u(t, \pi)=0
\end{array}\right.
$$

where the nonlinearity

$$
f(x, u)=a_{p}(x) u^{p}+O\left(u^{p+1}\right), \quad p \geq 2,
$$

vanishes at least quadratically at $u=0$.

Equation (1) is an infinite-dimensional Hamiltonian system possessing an elliptic equilibrium at $u=0$. Any solution $v=\sum_{j \geq 1} a_{j} \cos \left(j t+\theta_{j}\right) \sin (j x)$ of the linearized equation

$$
\left\{\begin{array}{l}
u_{t t}-u_{x x}=0 \\
u(t, 0)=u(t, \pi)=0
\end{array}\right.
$$

is $2 \pi$-periodic in time. For this reason, equation (1)-(2) is called a completely resonant PDE.

Question. Does there exist periodic solutions of the nonlinear equation (1)-(2) close to $u=0$ ?

2000 Mathematics Subject Classification: 35L05, 37K50, 58E05.

Supported by M.I.U.R. Variational Methods and Nonlinear Differential Equations.

The paper is in final form and no version of it will be published elsewhere. 
For finite-dimensional Hamiltonian systems, existence of periodic solutions close to a completely resonant elliptic equilibrium has been proved by Weinstein, Moser and Fadell-Rabinowitz. The proofs are based on the classical Lyapunov-Schmidt decomposition which splits the problem into (i) the range equation, solved through the standard implicit function theorem, (ii) the bifurcation equation, solved via variational arguments.

To extend these results for completely resonant Hamiltonian PDEs like (1)-(2), the main difficulties to be overcome are (i) a "small divisors problem" which prevents, in general, to use the implicit function theorem to solve the range equation; (ii) the presence of an infinite-dimensional bifurcation equation: which solutions $v$ of the linear equation (3) can be continued to solutions of the nonlinear equation (1)?

The "small divisors problem" (i) arises as follows. Since equation (1) is autonomous, the frequency $\omega$ of the periodic solution is not a priori fixed. We introduce $\omega$ as a free parameter looking for $2 \pi$-periodic solutions of

$$
\left\{\begin{array}{l}
\omega^{2} u_{t t}-u_{x x}+f(x, u)=0 \\
u(t, 0)=u(t, \pi)=0 .
\end{array}\right.
$$

The eigenvalues of the linear operator

$$
L_{\omega}:=\omega^{2} \partial_{t t}-\partial_{x x}
$$

in a space of functions $u(t, x), 2 \pi$-periodic in time and valued in $H_{0}^{1}(0, \pi)$ (because of Dirichlet boundary conditions) are

$$
\sigma\left(L_{\omega}\right) \equiv\left\{-\omega^{2} l^{2}+j^{2}, l \in \mathbf{Z}, j \geq 1\right\} .
$$

Therefore, for almost every $\omega \in \mathbf{R}$, the spectrum $\sigma\left(L_{\omega}\right)$ accumulates to 0 , implying that the inverse $L_{\omega}^{-1}$ is unbounded and the standard implicit function theorem is not applicable.

The first existence results of small amplitude periodic solutions of (1)-(2) have been obtained in [5] and in [20] (with periodic boundary conditions) for a zero measure set of frequencies such that $\sigma\left(L_{\omega}\right)$ does not accumulate to zero and so the small divisor problem (i) does not appear. The bifurcation equation (problem (ii)) is solved for $f=u^{3}+O\left(u^{5}\right)$ finding periodic solutions which are non-degenerate (i.e. the linearized equation has not non-trivial periodic solutions with the same period, see also Step 3 below).

In [8]-[9], for the same zero measure set of frequencies, existence and multiplicity of periodic solutions has been proved for any nonlinearity $f(u)$. The novelty of [8]-[9] was to solve the infinite-dimensional bifurcation equation via a variational principle at fixed frequency which, jointly with min-max arguments (the mountain pass theorem of Ambrosetti-Rabinowitz), enables to find solutions of (1) as critical points of the Lagrangian action functional.

We now want to consider the small divisors problem $(i)$ to find periodic solutions of (1)-(2) for asymptotically full measure sets of frequencies. Previous results in this direction have been obtained in [14] (for periodic spatial boundary conditions) and in [18] with the Lindsted series method, for $f=u^{3}+$ h.o.t.. Again the dominant term $u^{3}$ guarantees a non-degeneracy property. 
With the rescaling $u \rightarrow \delta u, \delta>0$, we obtain

$$
\left\{\begin{array}{l}
\omega^{2} u_{t t}-u_{x x}+\varepsilon g(\delta, x, u)=0 \\
u(t, 0)=u(t, \pi)=0
\end{array}\right.
$$

where

$$
\varepsilon:=\delta^{p-1} \quad \text { and } \quad g(\delta, x, u):=\frac{f(x, \delta u)}{\delta^{p}}=a_{p}(x) u^{p}+\delta a_{p+1}(x) u^{p+1}+\ldots
$$

We look for solutions of (5) in the Hilbert algebra $(\sigma>0, s>1 / 2)$

$X_{\sigma, s}:=\left\{u=\sum_{l \geq 0} \cos (l t) u_{l}(x): u_{l} \in H_{0}^{1}(0, \pi),\|u\|_{\sigma, s}^{2}:=\sum_{l \geq 0} e^{2 \sigma|l|}\left(l^{2 s}+1\right)\left\|u_{l}\right\|_{H^{1}}^{2}<+\infty\right\}$

of $2 \pi$-periodic, even, $\sigma$-analytic in time functions valued in $H_{0}^{1}(0, \pi)$ (we can look for even solutions because equation (1) is reversible).

The solutions of the linear equation (3) that belong to $H_{0}^{1}(\mathbf{T} \times(0, \pi), \mathbf{R})$ and are even in time form the infinite-dimensional linear space

$$
V:=\left\{v=\sum_{l \geq 1} \cos (l t) u_{l} \sin (l x): \sum_{l \geq 1} l^{2}\left|u_{l}\right|^{2}<+\infty\right\} .
$$

We implement the Lyapunov-Schmidt reduction according to the orthogonal decomposition

$$
X_{\sigma, s}=\left(V \cap X_{\sigma, s}\right) \oplus\left(W \cap X_{\sigma, s}\right)
$$

where

$$
W:=\left\{w=\sum_{l \geq 0} \cos (l t) w_{l}(x) \in X_{0, s} \text { such that } \int_{0}^{\pi} w_{l}(x) \sin (l x)=0\right\} .
$$

Projecting (1), setting $u=v+w, v \in V, w \in W$, and imposing the "frequency-amplitude" relation

$$
\frac{\omega^{2}-1}{2}=s^{*} \varepsilon
$$

with $s^{*}= \pm 1$ to be chosen later (see (10)), yields

$$
\begin{cases}-\Delta v=s^{*} \Pi_{V} g(\delta, x, v+w) & \text { bifurcation equation } \\ L_{\omega} w+\varepsilon \Pi_{W} g(\delta, x, v+w)=0 & \text { range equation }\end{cases}
$$

where $\Delta v:=v_{x x}+v_{t t}$ and $\Pi_{V}, \Pi_{W}$ denote the projectors respectively on $V$ and $W$.

Since $V$ is infinite-dimensional a serious difficulty arises in the application of the method of [17]: if $v \in V \cap X_{\sigma, s}$ then the solution $w(\delta, v)$ of the range equation, obtained with any Nash-Moser iteration scheme will have a lower regularity, e.g. $w(\delta, v) \in X_{\sigma / 2, s}$. Therefore, in solving next the bifurcation equation substituting $w=w(\delta, v)$, the best estimate we can obtain is $v \in V \cap X_{\sigma / 2, s+2}$ which makes the scheme incoherent. In [17] this problem does not arise since, if we deal with non-resonant PDEs like $u_{t t}-u_{x x}+a_{1}(x) u=$ $f(x, u)$, the bifurcation equation is finite-dimensional.

We overcome this difficulty thanks to a reduction to a finite-dimensional bifurcation equation on a subspace of $V$ of dimension $N$ independent of $\omega$. Let us decompose

$$
V=V_{1} \oplus V_{2}
$$


where

$$
\begin{cases}V_{1}:=\left\{v \in V: v(t, x)=\sum_{l=1}^{N} \cos (l t) u_{l} \sin (l x)\right\} & \text { "low Fourier modes" } \\ V_{2}:=\left\{v \in V: v(t, x)=\sum_{l>N} \cos (l t) u_{l} \sin (l x)\right\} & \text { "high Fourier modes". }\end{cases}
$$

By setting $v:=v_{1}+v_{2}, v_{1} \in V_{1}, v_{2} \in V_{2}$, system (7) is equivalent to

$$
\begin{cases}-\Delta v_{1}=s^{*} \Pi_{V_{1}} g\left(\delta, x, v_{1}+v_{2}+w\right) & (Q 1) \\ -\Delta v_{2}=s^{*} \Pi_{V_{2}} g\left(\delta, x, v_{1}+v_{2}+w\right) & (Q 2) \\ L_{\omega} w+\varepsilon \Pi_{W} g\left(\delta, x, v_{1}+v_{2}+w\right)=0 & \text { range equation }\end{cases}
$$

where $\Pi_{V_{i}}: X_{\sigma, s} \rightarrow V_{i}(i=1,2)$ denote the projectors on $V_{i}$.

Step 1: Solution of the (Q2)-equation. Thanks to the compactness of $(-\Delta)^{-1}$, the solution $v_{2}\left(\delta, v_{1}, w\right) \in V_{2} \cap X_{\sigma, s+2}$ of the (Q2)-equation is found by a contraction mapping argument provided $N$ is large enough and $0<\sigma \leq \bar{\sigma}$ is small enough, depending only on the nonlinearity $f$. To clarify this point note that the 0 th order bifurcation equation

$$
-\Delta v=s^{*} \Pi_{V}\left(a_{p}(x) v^{p}\right)
$$

(obtained by setting $\delta=0$ in the bifurcation equation) is the Euler Lagrange equation of

$$
\Phi_{0}(v):=\frac{\|v\|_{H^{1}}^{2}}{2}-s^{*} \int_{\Omega} a_{p}(x) \frac{v^{p+1}}{p+1}, \quad \Omega:=\mathbf{T} \times(0, \pi),
$$

where $\|v\|_{H^{1}}^{2}=\int_{\Omega} v_{t}^{2}+v_{x}^{2}$. If we take

$$
s^{*}= \begin{cases}1 & \text { if } \exists v \in V \text { such that } \int_{\Omega} a_{p}(x) v^{p+1}>0 \\ -1 & \text { if } \exists v \in V \text { such that } \int_{\Omega} a_{p}(x) v^{p+1}<0\end{cases}
$$

$\Phi_{0}$ possesses a non-trivial mountain pass critical set $\mathcal{K}$ which is compact for the $H^{1}$-topology [8]. $N$ must be chosen large enough depending only on $\mathcal{K}$ : heuristically $N$ must be taken large enough so that the majority of the $H^{1}$-norm of the elements of $\mathcal{K}$ is "concentrated" on the first $N$ Fourier modes.

Step 2: Solution of the range equation. We find next a solution $w\left(\delta, v_{1}\right) \in W \cap X_{\bar{\sigma} / 2, s}$ of the range equation by means of a Nash-Moser Implicit Function Theorem for $\left(\delta, v_{1}\right)$ belonging to some Cantor-like set $B_{\infty}$ of parameters. To understand how the Cantor set $B_{\infty}$ arises, we recall that the core of any Nash-Moser convergence method (based on Newton's iteration scheme) is the proof of the invertibility of the linearized operators obtained at a given stage of the Nash-Moser iteration. Restrictions on $\left(\delta, v_{1}\right)$ arise imposing that their eigenvalues (which are dense on $\mathbf{R}$ as the spectrum $\sigma\left(L_{\omega}\right)$ in (4)) are sufficiently different from zero to control the norm of their inverses and to obtain the convergence of the iterative scheme.

Our approach to invert the linearized operators is different than in [17] and works also for not odd nonlinearities $f$ with low spatial regularity, unlike [17] works only for $f$ odd and analytic in $(x, u)$.

Step 3: solution of the (Q1)-equation. Every solution $\bar{v} \in V$ of the 0th order bifurcation equation (9) which is non-degenerate, i.e. $\operatorname{Ker} \Phi_{0}^{\prime \prime}(\bar{v})=\{0\}$, can be continued to 
a solution of (1) for an asymptotically full measure set of frequencies. Indeed, under this non-degeneracy condition, there is a $C^{\infty}$-curve $\delta \mapsto v_{1}(\delta) \in V_{1}, v_{1}(0)=\Pi_{V_{1}} \bar{v}$, of solutions of the finite-dimensional $(Q 1)$-equation which intersects transversally - and so in a asymptotically full measure set - the Cantor set $B_{\infty}$ where also the range equation was solved. This non-degeneracy condition (analogue to the KAM-Arnold condition) has been verified in [10], [2] for nonlinearities like in (11) and it is difficult to check, if ever true, for a general nonlinear term $a_{p}(x) u^{p}$ for any $p$.

Finally we prove

TheOREM 1.1 ([10]). Assume that

$$
f(x, u)= \begin{cases}a_{2} u^{2}+\sum_{k \geq 4} a_{k}(x) u^{k}, & a_{2} \neq 0 \\ a_{3}(x) u^{3}+\sum_{k \geq 4} a_{k}(x) u^{k}, & \left\langle a_{3}\right\rangle:=\pi^{-1} \int_{0}^{\pi} a_{3}(x) \neq 0 \\ a_{4} u^{4}+\sum_{k \geq 8} a_{k}(x) u^{k}, & a_{4} \neq 0\end{cases}
$$

where $a_{k}(x) \in H^{1}(0, \pi)$ satisfy $\sum_{k}\left\|a_{k}\right\|_{H^{1}} \rho^{k}<\infty$ for some $\rho>0$. Then, $s>1 / 2$ being given, there exist $\delta_{0}>0, \bar{\sigma}>0$ and $a C^{\infty}$-curve $\left[0, \delta_{0}\right) \ni \delta \mapsto u(\delta) \in X_{\bar{\sigma} / 2, s}$ with the following properties:

(i) $\|u(\delta)-\delta \bar{v}\|_{\bar{\sigma} / 2, s}=O\left(\delta^{2}\right)$ for some $\bar{v} \in V \cap X_{\bar{\sigma}, s}, \bar{v} \neq 0$;

(ii) there exists a Cantor set $\mathcal{C} \subset\left[0, \delta_{0}\right)$ of asymptotically full measure, i.e.

$$
\lim _{\eta \rightarrow 0^{+}} \frac{\operatorname{meas}(\mathcal{C} \cap(0, \eta))}{\eta}=1,
$$

such that, for each $\delta \in \mathcal{C}, u(\delta)(\omega(\delta) t, x)$ is a $2 \pi / \omega(\delta)$-periodic solution of (1) with

$$
\omega(\delta)=\left\{\begin{array}{l}
\sqrt{1-2 \delta^{2}} \\
\sqrt{1+2 \delta^{2} \operatorname{sign}\left\langle a_{3}\right\rangle} \\
\sqrt{1-2 \delta^{6}}
\end{array}\right.
$$

We want next to relax the non-degeneracy condition finding solutions of the finitedimensional bifurcation equation through variational methods. This allows to deal with more general nonlinearities.

The big difficulty is that, in the degenerate case, $v_{1}(\delta)$ could vary in a highly irregular way as $\delta \rightarrow 0$, and could belong, for each $\delta$, to the complementary of the Cantor set $B_{\infty}$ where the range equation was solved (actually $B_{\infty}^{c}$ is even arcwise connected!). This is the common difficulty in applying variational methods in a problem with small divisors.

To have a sufficiently good control on how $v_{1}(\delta)$ varies with $\delta$ (we prove in [11] that it is sufficient that $\delta \mapsto \delta^{m} v_{1}(\delta)$ is a BV function for some $m>0$ ) we use ideas related to the Struwe "monotonicity method" [25] for families of functionals possessing the mountain pass geometry and we get the BV dependence for parameter depending nonlinearities (arbitrarily close to $a_{p}(x) u^{p}$ )

$$
f(x, u, \lambda)=a_{p}(x) u^{p}+\sum_{i=1}^{M} \lambda_{i} b_{i}(x) u^{q_{i}}+r(x, u), \quad q_{i}>p,
$$

where $r(x, u):=\sum_{k>p} a_{k}(x) u^{k}$ satisfies $\|r\|_{\rho}:=\sum_{k>p}\left\|a_{k}\right\|_{H^{1}} \rho^{k}<\infty$ for some $\rho>0$. 
TheOREM $1.2([11])$. For any $\bar{q}>p$ there exist $M$ integer exponents $\left({ }^{1}\right) \bar{q} \leq q_{1} \leq \ldots \leq$ $q_{M}$ and coefficients $b_{1}, \ldots, b_{M} \in H^{1}(0, \pi)$ such that, for any $r(x, u)=\sum_{k>p} r_{k}(x) u^{k}$ with $\|r\|_{\rho}<1$, for almost every parameter $\lambda=\left(\lambda_{1}, \ldots, \lambda_{M}\right),|\lambda| \leq 1$, equation (1) with nonlinearity $f(\lambda, x, u)$ like in (12) possesses small amplitude periodic solutions for an asymptotically full measure Cantor set of frequencies $\omega$ close to 1.

We remark that, since $q_{i}>p$, the nonlinearities $\lambda_{i} b_{i}(x) u^{q_{i}}$ (as well as $r(x, u)=$ $O\left(u^{p+1}\right)$ ) do not change the 0th-order bifurcation equation (9), which keeps being possibly degenerate. Actually, since $q_{i}>\bar{q}$ can be arbitrarily large, we are adding arbitrarily small corrections $b_{i}(x) u^{q_{i}}=o\left(u^{p}\right)$ for $u \rightarrow 0$. Moreover, given $a_{p}(x) u^{p}, b_{i}(x) u^{q_{i}}$, Theorem 1.2 holds for any nonlinear term $\|r\|_{\rho}<1$, only with a change of the full measure set of parameters $\lambda$. Furthermore the finite set of $b_{i}(x), q_{i}$ can be taken the same for $a_{p}$ in a neighbourhood of a given $\bar{a}_{p}$ in $H^{1}(0, \pi)$. For these reasons Theorem 1.2 can be interpreted as a genericity result in the sense of "Lebesgue measure" (it is much stronger than a density result), in the vector space of nonlinearities

$\mathcal{F}:=\left\{f(x, u):=a_{p}(x) u^{p}+\sum_{k>p} f_{k}(x) u^{k}, f_{k}(x) \in H^{1}(0, \pi),\|f\|_{\rho}:=\sum_{k \geq p}\left\|f_{k}\right\|_{H^{1}} \rho^{k}<\infty\right\}$, see [11] for details.

2. Forced vibrations. We consider now the existence problem of periodic solutions for completely resonant forced wave equations like

$$
\left\{\begin{array}{l}
u_{t t}-u_{x x}=\varepsilon f(t, x, u) \\
u(t, 0)=u(t, \pi)=0
\end{array}\right.
$$

where the nonlinear forcing term

$$
f(t+T, x, u)=f(t, x, u)
$$

is $T$-periodic in time and $\varepsilon$ is a small parameter. Hence we have to look for $T$-periodic solutions of (13).

Clearly it makes a big difference if the forcing frequency $\omega:=T / 2 \pi$ satisfies

$$
\text { (i) } \omega \in \mathbf{Q} \quad \text { or } \quad \text { (ii) } \omega \in \mathbf{R} \backslash \mathbf{Q} \text {. }
$$

The second case (ii) leads to a small divisor problem similar to the one considered in the previous section, see e.g. [21], [3] and references therein. On the contrary, here we investigate the case (i) which leads to completely different phenomena, for example when

$$
T=2 \pi \text {. }
$$

The main difficulty for proving existence of solutions of (13)-(14) for $\varepsilon \neq 0$ is now to solve the infinite-dimensional bifurcation equation which exhibits an intrinsic lack of compactness (while the range equation is now easily solved).

The first breakthrough regarding problem (13)-(14) was achieved by Rabinowitz in [23] where existence and regularity of solutions was proved for strongly monotone

$\left.{ }^{1}\right) M$ depends in general on $\bar{q}$. Furthermore we could choose $\bar{q}<q_{1}<\ldots<q_{M}$ with strict inequality. 
nonlinearities $\left(\partial_{u} f\right)(t, x, u) \geq \beta>0$. Subsequently, Rabinowitz [24] was able to prove existence of weak solutions of (13) for weakly monotone nonlinearities like $f(t, x, u)=$ $u^{2 k+1}+G(t, x, u)$ where $G\left(t, x, u_{2}\right) \geq G\left(t, x, u_{1}\right)$ if $u_{2} \geq u_{1}$. Concerning regularity, Brézis and Nirenberg [15] proved - but only for strongly monotone nonlinearities - that any $L^{\infty}$. solution of (13) is smooth, even in the non-perturbative case $\varepsilon=1$, whenever $f$ is smooth.

Without monotonicity, Willem [26], Hofer [19] and Coron [16] have considered $f=g(u)+h(t, x), \varepsilon=1$, with $g(u)$ satisfying suitable linear growth conditions. Existence of weak solutions is proved, in [26], [19], for a set of $h$ dense in $L^{2}$, although without explicit criteria that characterize such $h$. The infinite-dimensional bifurcation problem is overcome by assuming non-resonance hypothesis between the asymptotic behaviour of $g(u)$ and the spectrum of the D'Alembertian. On the other side, Coron [16] finds weak solutions assuming additional symmetries and restricting to subspaces where the kernel of the d'Alembertian reduces to 0 .

In [6]-[7] existence and regularity of solutions of (13) have been proved for a large class of non-monotone forcing terms $f(t, x, u)$. Let us present these results. We look for solutions $u$ of (13) in the Banach space

$$
E:=H^{1}(\Omega) \cap C_{0}^{1 / 2}(\bar{\Omega}), \quad \Omega:=\mathbf{T} \times(0, \pi)
$$

where $C_{0}^{1 / 2}(\bar{\Omega})$ is the space of the $1 / 2$-Hölder continuous functions which are zero at $x=0, x=\pi$, endowed with its natural norm $\|u\|_{E}:=\|u\|_{H^{1}(\Omega)}+\|u\|_{C^{1 / 2}(\bar{\Omega})}$.

Critical points of the Lagrangian action functional $\Psi \in C^{1}(E, \mathbf{R})$

$$
\Psi(u):=\int_{\Omega}\left[\frac{u_{t}^{2}}{2}-\frac{u_{x}^{2}}{2}+\varepsilon F(t, x, u)\right] d t d x
$$

where $F(t, x, u):=\int_{0}^{u} f(t, x, \xi) d \xi$ are weak solutions of (13).

For $\varepsilon=0$, the critical points of $\Psi$ in $E$ form the subspace $V=N \cap H^{1}(\Omega)$ where

$$
N:=\left\{v=\hat{v}(t+x)-\hat{v}(t-x): \hat{v} \in L^{2}(\mathbf{T}) \text { and } \int_{0}^{2 \pi} \hat{v}(s) d s=0\right\} .
$$

Here we represent the solutions of (3) in D'Alembertian form as a linear superposition of two waves travelling in opposite directions with the same speed (it is equivalent to the Fourier representation in (6)).

Define $N^{\perp}:=\left\{h \in L^{2}(\Omega): \int_{\Omega} h v=0 \forall v \in N\right\}$.

ThEOREM $2.1([6]-[7])$. Let $f(t, x, u)=\beta u^{2 k}+h(t, x)$ with $h \in N^{\perp}$ and $h(t, x)>0$ (or $h(t, x)<0)$ a.e. in $\Omega$. Then

(i) (Existence) For $\varepsilon$ small enough, there is at least one weak solution $u \in E$ of (13) with $\|u\|_{E} \leq C|\varepsilon|$.

(ii) (Regularity) If $h \in H^{j}(\Omega) \cap C^{j-1}(\bar{\Omega})$ then $u \in H^{j+1}(\Omega) \cap C_{0}^{j}(\bar{\Omega})$.

We deal with more general nonlinearities in Theorems 2, 3 of [6]. We remark, however, that the assumption $h \in N^{\perp}$ is not of technical nature: if $h \notin N^{\perp}$, periodic solutions of (13) do not exist in any fixed ball $\left\{\|u\|_{L^{\infty}} \leq R\right\}$ for $\varepsilon$ small.

REMARK 2.1 (Multiplicity [6]). There exist infinitely many $h \in N^{\perp}$ for which equation (13) with $f=\beta u^{2 k}+h(t, x)$ has at least 3 solutions. 
To find critical points of $\Psi: E \rightarrow \mathbf{R}$ we perform a Lyapunov-Schmidt reduction, decomposing

$$
E=V \oplus W
$$

where $W:=E \cap N^{\perp}$. Setting $u=v+w, v \in V, w \in W$, and denoting by $\Pi_{N}$ and $\Pi_{N^{\perp}}$ the projectors onto $N$ and $N^{\perp}$ respectively, we see that problem (13) is equivalent to

$$
\begin{cases}\Pi_{N} f(v+w)=0 & \text { bifurcation equation } \\ w=\varepsilon L^{-1} \Pi_{N^{\perp}} f(v+w) & \text { range equation }\end{cases}
$$

where $L^{-1}: N^{\perp} \rightarrow N^{\perp}$ denotes the inverse of the D'Alembertian operator.

We find a solution $w:=w(v, \varepsilon) \in W$ of the range equation satisfying $\|w(v, \varepsilon)\|_{E}$ $=O(\varepsilon)$ by the standard implicit function theorem since $L^{-1}$ acting on $W$ is a compact operator for the assumption $T=2 \pi$ (actually $\left\|L^{-1} f\right\|_{E} \leq C\|f\|_{L^{2}}, \forall f \in L^{2}$, and this motivates the choice of the space $E$ in $(15))$.

There remains the infinite-dimensional bifurcation equation

$$
\Pi_{N} f(v+w(v, \varepsilon))=0
$$

which is the Euler-Lagrange equation of the reduced Lagrangian action functional

$$
\Phi: V \mapsto \mathbf{R} \quad \Phi(v):=\Psi(v+w(v, \varepsilon)) .
$$

Since $\Phi$ lacks compactness properties, we cannot rely on critical point theory, unlike the autonomous case considered in the previous section (where the term $\|v\|_{H^{1}}^{2}$ is present). We attempt to minimize $\Phi$, but we do not try to apply the direct methods of the calculus of variations because $\Phi$ will not be convex (being $f$ non-monotone). We minimize $\Phi$ constrained in $\bar{B}_{R}:=\left\{\|v\|_{H^{1}} \leq R\right\}$. By standard compactness arguments $\Phi$ attains minimum at, say, $\bar{v} \in \bar{B}_{R}$ and we can only conclude the variational inequality

$$
D_{v} \Phi(\bar{v})[\varphi]=\int_{\Omega} f(\bar{v}+w(\bar{v}, \varepsilon)) \varphi \leq 0
$$

for any admissible variation $\varphi \in V$, i.e. such that $\bar{v}+\theta \varphi \in B_{R}, \forall \theta<0$ sufficiently small.

The heart of the existence proof of Theorem 2.1 is to obtain, choosing suitable admissible variations the a priori estimate $\|\bar{v}\|_{H^{1}}<R^{*}$ for some $R^{*}>0$, i.e. to show that $\bar{v}$ is an inner minimum point of $\Phi$ in $B_{R^{*}}$ (the strong monotonicity assumption $\left(\partial_{u} f\right)(t, x, u) \geq \beta>0$ would allow here to get such a priori estimate by arguments similar to [23]).

To understand the difficulty, let consider the particular nonlinearity $f=u^{2}+h(t, x)$. The even term $u^{2}$ does not give any contribution into the variational inequality (16) for $\varepsilon=0$ because

$$
\int_{\Omega}\left(\bar{v}^{2}+h(t, x)\right) \varphi=0, \quad \forall \varphi \in V
$$

since $h \in N^{\perp}$ and $\int_{\Omega} \bar{v}^{2} \varphi \equiv 0$. Therefore we have to develop (16) at higher orders in $\varepsilon$, obtaining

$$
0 \geq \int_{\Omega} 2 \bar{v} \varphi w(\bar{v}, \varepsilon)+O\left(w^{2}(\bar{v}, \varepsilon)\right)=\int_{\Omega} \varepsilon 2 \bar{v} \varphi L^{-1}\left(h+\bar{v}^{2}\right)+O\left(\varepsilon^{2}\right)
$$

because $w(\bar{v}, \varepsilon)=\varepsilon L^{-1}\left(\bar{v}^{2}+h\right)+o(\varepsilon)$ (by $\left.\bar{v}^{2}, h \in N^{\perp}\right)$. 
We now sketch how (17) allows to prove an $L^{2}$-estimate for $\bar{v}$. Inserting $\varphi:=\bar{v}$ in (17) we get

$$
\int_{\Omega} H \bar{v}^{2}+\bar{v}^{2} L^{-1} \bar{v}^{2} \leq O(\varepsilon)
$$

where $H$ is a weak solution of $\left(\partial_{t t}-\partial_{x x}\right) H=h$ which verifies $H(t, x)>0$ in $\Omega$ ( $H$ exists by the "maximum principle" proposition of [6]). The first term in (18) satisfies the coercivity inequality

$$
\int_{\Omega} H v^{2} \geq c(H) \int_{\Omega} v^{2}, \quad \forall v \in V
$$

for some $c(H)>0$, which is not trivial because $H$ vanishes at the boundary. Indeed, (19) holds for the travelling wave form $v=\hat{v}(t+x)-\hat{v}(t-x)$ of the functions of $V$. The second term $\int_{\Omega} \bar{v}^{2} L^{-1} \bar{v}^{2} \geq 0$ (by the "maximum principle") and (18)-(19) provide $\|\bar{v}\|_{L^{2}}=O(\varepsilon)$. Next, the $L^{\infty}$ and $H^{1}$-estimate (independent of $R$ ) for $\bar{v}$ are obtained inserting admissible variations $\varphi$ inspired to [23] into (17) and using inequalities similar to $(19)$.

The regularity of the solution $u$ in Theorem 2.1-(ii) - not obvious for non-monotone nonlinearities - is proved using similar techniques (inspired, as in [23], to elliptic regularity theory).

3. Quasi-periodic solutions. We consider now the existence problem of small amplitude quasi-periodic solutions for completely resonant forced wave equations like

$$
\left\{\begin{array}{l}
u_{t t}-u_{x x}+f\left(\omega_{1} t, u\right)=0 \\
u(t, x)=u(t, x+2 \pi)
\end{array}\right.
$$

where the nonlinear forcing term

$$
f\left(\omega_{1} t, u\right)=a\left(\omega_{1} t\right) u^{2 d-1}+O\left(u^{2 d}\right), \quad d>1,
$$

is $2 \pi / \omega_{1}$-periodic in time. The main difficulty is (jointly to a small divisor problem) to understand from which solutions of the linear equation (3) quasi-periodic solutions branchoff, since all the solutions of (3) are $2 \pi$-periodic. For completely resonant autonomous PDEs, existence of quasi-periodic solutions with 2-frequencies has been recently obtained in [22], [1] for $f=u^{3}+O\left(u^{5}\right)$.

In [12]-[13] existence of quasi-periodic solutions with two frequencies $\omega_{1}, \omega_{2}$ has been proved for the completely resonant forced equation (20) in both the cases

$$
\text { (i) } \omega_{1} \in \mathbf{Q} \quad \text { (ii) } \omega_{1} \in \mathbf{R} \backslash \mathbf{Q} \text {. }
$$

We present below only the case (i) when $\omega_{1}$ enters in resonance with the linear frequency 1. We look for quasi-periodic solutions of (20) of the form

$$
u(t, x)=U\left(\omega_{1} t, \omega_{2} t+x\right)
$$

with frequencies $\left(\omega_{1}, \omega_{2}\right)=\left(\omega_{1}, 1+\varepsilon\right)$ and $U$ in the Banach algebra

$$
\mathcal{H}_{\sigma, s}:=\left\{U(\varphi)=\sum_{l \in \mathbf{Z}^{2}} \hat{U}_{l} e^{i l \cdot \varphi}: \hat{U}_{l}^{*}=\hat{U}_{-l} \text { and }|U|_{\sigma, s}:=\sum_{l \in \mathbf{Z}^{2}}\left|\hat{U}_{l}\right| e^{\left|l_{2}\right| \sigma}\left[l_{1}\right]^{s}<+\infty\right\}
$$


where $\left[l_{1}\right]:=\max \left\{\left|l_{1}\right|, 1\right\}, \sigma>0, s \geq 0$. We assume that

$$
f\left(\varphi_{1}, u\right)=\sum_{k=2 d-1}^{\infty} a_{k}\left(\varphi_{1}\right) u^{k}, d \in \mathbf{N}^{+}, d>1 \text { and } a_{k}\left(\varphi_{1}\right) \in H^{1}(\mathbf{T}) \text { verify }
$$

for some $r>0, \sum_{k=2 d-1}^{\infty}\left|a_{k}\right|_{H^{1}} r^{k}<\infty$. The function $f\left(\varphi_{1}, u\right)$ is not

identically constant in $\varphi_{1}$.

Theorem 3.1 ([12]-[13]). Let $\omega_{1}=n / m \in \mathbf{Q}$. Assume $(\mathrm{H})$ and $a_{2 d-1}\left(\varphi_{1}\right) \neq 0$ for each $\varphi_{1} \in \mathbf{T}$. Let $\mathcal{B}_{\gamma}$ be the uncountable zero-measure Cantor set

$$
\mathcal{B}_{\gamma}:=\left\{\varepsilon \in\left(-\varepsilon_{0}, \varepsilon_{0}\right):\left|l_{1}+\varepsilon l_{2}\right|>\frac{\gamma}{\left|l_{2}\right|} \quad \forall l_{1}, l_{2} \in \mathbf{Z} \backslash\{0\}\right\}
$$

where $0<\gamma<1 / 6$. There exist $\bar{\sigma}>0, \bar{s}>2, \bar{\varepsilon}>0$, such that $|\varepsilon| \gamma^{-1} \leq \bar{\varepsilon} / m^{2}$ for all $\varepsilon \in \mathcal{B}_{\gamma}$, equation (20) admits a small amplitude quasi-periodic solution $u(t, x)=$ $U\left(\omega_{1} t, x+\omega_{2} t\right), U(\varphi) \in \mathcal{H}_{\bar{\sigma}, \bar{s}}$, with two frequencies $\left(\omega_{1}, \omega_{2}\right)=(n / m, 1+\varepsilon)$, of the form $u(t, x)=|\varepsilon|^{1 /(2(d-1))}\left[q_{+}(x+(1+\varepsilon) t)+q_{-}((1-\varepsilon) n t-n x)\right]+O\left(\frac{m^{2}}{\gamma \omega_{1}^{3}}|\varepsilon|^{(2 d-1) /(2(d-1))}\right)$ where $q_{+}, q_{-}$are suitable $2 \pi$-periodic $\bar{\sigma}$-analytic functions.

At the first order the quasi-periodic solution $u$ is the linear superposition of two waves travelling in opposite directions (in general, both $q_{+}, q_{-}$are non-trivial) with speeds $1+\varepsilon$ and $-1+\varepsilon$.

As we said, the main difficulty to prove Theorem 3.1 is to find out from which solutions of the linear equation (3) quasi-periodic solutions of (20) branch-off, i.e. to find $q_{+}, q_{-}$. This requires to solve an infinite-dimensional bifurcation equation which cannot be solved in general by o.d.e. techniques (it is a system of integro-differential equations). However, exploiting the variational nature of (20) the bifurcation problem reduces - like in the previous sections - to find critical points of a reduced Lagrangian action functional. In this case such a functional possesses the infinite-dimensional linking geometry of the theorem of Benci-Rabinowitz. A suitable variation of this theorem (required by the fact that the reduced functional is defined only in a neighbourhood of the origin) enables to find out the (analytic) bifurcation solutions $q_{+}, q_{-}$.

If $\omega_{1}$ is irrational (case (ii)) the bifurcation of quasi-periodic solutions looks quite different, see Theorem B of [12]. Finally we remark that, imposing $\omega_{1} / \omega_{2}=$ $\omega_{1} /(1+\varepsilon) \in \mathbf{Q}$, we obtain also periodic solutions with large minimal period which are reminiscent, in this completely resonant context, of the Birkhoff-Lewis periodic orbits accumulating at the origin, see [4].

\section{References}

[1] P. Baldi, Quasi-periodic solutions of the equation $v_{t t}-v_{x x}+v^{3}=f(v)$, Discrete Contin. Dyn. Syst. 15 (2006), 883-903.

[2] P. Baldi, M. Berti, Periodic solutions of nonlinear wave equations for asymptotically full measure sets of frequencies, Atti Accad. Naz. Lincei Cl. Sci. Fis. Mat. Natur. Rend. Lincei (9) Math. Appl. 17 (2006), 257-277. 
[3] P. Baldi, M. Berti, Forced vibrations of a material string, preprint 2006.

[4] D. Bambusi, M. Berti, A Birkhoff-Lewis type theorem for some Hamiltonian PDEs, SIAM J. Math. Anal. 37 (2005), 83-102.

[5] D. Bambusi, S. Paleari, Families of periodic solutions of resonant PDEs, J. Nonlinear Sci. 11 (2001), 69-87.

[6] M. Berti, L. Biasco, Forced vibrations of wave equations with non-monotone nonlinearities, Ann. Inst. H. Poincaré Anal. Non Linéaire 23 (2006), 439-474.

[7] M. Berti, L. Biasco, Periodic solutions of nonlinear wave equations with non-monotone forcing term, Atti Accad. Naz. Lincei Cl. Sci. Fis. Mat. Natur. Rend. Lincei (9) Math. Appl. 16 (2005), 117-124.

[8] M. Berti, P. Bolle, Periodic solutions of nonlinear wave equations with general nonlinearities, Comm. Math. Phys. 243 (2003), 315-328.

[9] M. Berti, P. Bolle, Multiplicity of periodic solutions of nonlinear wave equations, Nonlinear Anal. 56 (2004), 1011-1046.

[10] M. Berti, P. Bolle, Cantor families of periodic solutions for completely resonant nonlinear wave equations, Duke Math. J. 134 (2006), 359-419.

[11] M. Berti, P. Bolle, Cantor families of periodic solutions for wave equations via a variational principle, preprint 2006.

[12] M. Berti, M. Procesi, Quasi-periodic solutions of completely resonant forced wave equations, Comm. Partial Differential Equations 31 (2006), 959-985.

[13] M. Berti, M. Procesi, Quasi-periodic oscillations for wave equations with periodic forcing, Atti Accad. Naz. Lincei Cl. Sci. Fis. Mat. Natur. Rend. Lincei (9) Math. Appl. 16 (2005), 109-116.

[14] J. Bourgain, Periodic solutions of nonlinear wave equations, in: Harmonic Analysis and Partial Differential Equations, Chicago Lectures in Math., Univ. Chicago Press, Chicago 1999, 69-97.

[15] H. Brézis, L. Nirenberg, Forced vibrations for a nonlinear wave equation, Comm. Pure Appl. Math. 31 (1978), 1-30.

[16] J.-M. Coron, Periodic solutions of a nonlinear wave equation without assumption of monotonicity, Math. Ann. 262 (1983), 273-285.

[17] W. Craig, C. E. Wayne, Newton's method and periodic solutions of nonlinear wave equations, Comm. Pure Appl. Math. 46 (1993), 1409-1498.

[18] G. Gentile, V. Mastropietro, M. Procesi, Periodic solutions for completely resonant nonlinear wave equations, Comm. Math. Phys. 256 (2005), 437-490.

[19] H. Hofer, On the range of a wave operator with non-monotone nonlinearity, Math. Nachr. 106 (1982), 327-340.

[20] B. V. Lidskiü, E. I. Shul'man, Periodic solutions of the equation $u_{t t}-u_{x x}+u^{3}=0$, Funktsional. Anal. i Prilozhen. 22:4 (1988), 88-89 (in Russian); English transl.: Funct. Anal. Appl. 22 (1988), 332-333.

[21] P. I. Plotnikov, L. N. Yungerman, Periodic solutions of a weakly nonlinear wave equation with an irrational relation of period to interval length, Differentsial'nye Uravneniya 24 (1988), 1599-1607; English transl.: Differential Equations 24 (1988), 1059-1065.

[22] M. Procesi, Quasi-periodic solutions for completely resonant nonlinear wave equations in 1D and 2D, Discrete Contin. Dyn. Syst. 13 (2005), 541-552.

[23] P. H. Rabinowitz, Periodic solutions of nonlinear hyperbolic partial differential equations, Comm. Pure Appl. Math. 20 (1967), 145-205. 
[24] P. H. Rabinowitz, Time periodic solutions of nonlinear wave equations, Manuscripta Math. 5 (1971), 165-194.

[25] M. Struwe, The existence of surfaces of constant mean curvature with free boundaries, Acta Math. 160 (1988), 19-64.

[26] M. Willem, Density of the range of potential operators, Proc. Amer. Math. Soc. 83 (1981), $341-344$. 